\title{
Repercussões psicossomáticas na Epiderme Humana
}

\author{
Marianna Leite Barroso ${ }^{1}$; Maria Ayrlles Macêdo ${ }^{2}$
}

Resumo: Na atualidade, parece haver um incremento quanto aos estudos que envolvem uma integração entre aspectos biológicos e psicológicos, mais especificamente entre corpo e estado psíquico. O presente estudo pretende analisar, através de revisão teórica, achados teóricos da Psicologia associados a dermatologia, de maneira a permitir reflexão crítica a respeito do tema e, vislumbrar seus novos rumos. A Psicologia quando associada a dermatologia, compõe um ramo das ciências que estuda problemas dermatológicos, que são causados e/ou influenciados por fatores psicológicos. Sabe-se que há uma comunicação entre o sistema nervoso, que torna a pele altamente sensível às emoções, independente da nossa consciência. Dessa forma, a pele é muitas vezes uma expressão dos nossos sentimentos, mesmo quando não estamos cientes de tal fato. Conclui-se que ao tratarmos sobre as doenças de pele, é preciso considerar também o ser humano como um ser integral, onde aspectos sociais, psicológicos e biológicos estão em constante interação.

Palavras-chave: Pele, Psicossomática, Dermatologia.

\section{Repercussions psychosomatic in Human epidermi}

\begin{abstract}
Currently, it seems to be an increase in the studies involving the integration of biological and psychological aspects, specifically between body and mental state. This study aims to analyze, through literature review, theoretical findings related to Psychology associate with dermatology, in order to allow critical reflection on the subject, and envision its new directions. There is an understanding that Psychology associate with dermatology is the branch that studies dermatological problems, which are caused and / or influenced by psychological factors. It is known that there is a communication between the nervous system, which makes the skin highly sensitive to emotions, regardless of our consciousness. Thus, the skin is often an expression of our feelings, even when we are not aware of this fact. We conclude that at the treatment of skin diseases, one must also consider the human being as a whole, where social, psychological and biological aspects are in constant interaction.
\end{abstract}

Keywords: Skin, Psychosomatics, Dermatology.

\section{Introdução}

A cultura oriental, sempre apresentou indícios de uma associação entre a mente e o corpo, nos reportando a uma noção de indissociabilidade entre esses. Isto é, a noção de uma integração entre corpo e mente. Na Índia, há anos que a Psicologia ocupa um espaço importante dentro dos estudos da medicina, ajudando na compreensão de muitas doenças (AZAMBUJA, 2000).

\footnotetext{
${ }^{1}$ Graduação em Enfermagem pela Faculdade de Enfermagem São Vicente de Paula. Mestranda em Saúde pública pela Universidade Tecnológica Intercontinental - UTIC/PY. Especialista em Enfermagem Obstétrica pelo Centro de Aperfeiçoamento Profissional LTDA CEFAPP (Em Andamento). Especialista em Saúde Pública pela Faculdade São Francisco da Paraíba FASP. Especialista em Saúde da Família pela Faculdade Nossa Senhora de Lourdes- FNSL Centro Integrado de Tecnologia e pesquisa. Docente efetiva do Curso Superior de Enfermagem pela Faculdade São Francisco da Paraiba - FASP. Enfermeira Obstétrica da Maternidade Dr. Deodato Cartaxo, no Hospital Regional de Cajazeiras e Enfermeira da Unidade de Saúde da Família Higino Dias Moreira pela secretária Municipal de Saúde CajazeirasPB. E-mail: mariannaleite_@hotmail.com

${ }^{2}$ Psicóloga Faculdade Leão Sampaio. Pós-Graduada pela Escola de Saúde Pública do Ceara na modalidade de Residência em Saúde da

Família.Atualmente Psicologa do NASF do município de Granjeiro-CE. Email: ayrllesmacedo@ hotmail.com;
} 
Nestes estudos, não caberia pois, uma visão de homem fragmentada, ou um homem dissociado do seu meio ou da sua psique.

A noção de um ser humano, como algo além do seu corpo físico é antiga. Para Silva (2002), tal noção surgiu pela necessidade de confrontar a morte, suas ansiedades e temores associados, dessa forma, deixando o homem mais atento e desperto para os mistérios da alma e para as forças externas ao corpo.

As doenças psicossomáticas dermatológicas são condições que envolvem uma interação entre a mente e pele. Koo e Lebwohl (2001) endossam essa ideia, indicando que as desordens da pele ocorrem de três maneiras: a) desordens psicofisiológicas, b) desordens psiquiátricas primárias e c) desordens psiquiátricas secundárias.

Na década de 1980, autores como Bechelli e Curban (1988) já abordavam tais questões, sobre a possibilidade de uma integração entre a mente e o corpo, em relação à dermatologia. Eles mencionaram que, as alterações cutâneas poderiam sim estarem associadas a diversos distúrbios psíquicos, enquanto causa e efeito.

A relação entre a mente e a pele parece envolver todos os elementos, que são subjetivos, e que estão presentes em nossa personalidade. Portanto, emoções, sentimentos, agressividade e fantasias, fazem parte deste elenco e, conforme suas intensidades, eles refletem-se na pele, alimentando uma possível enfermidade cutânea (MÜLLER, 2001).

Hoje, uma nova Medicina vem despontando no cenário global, baseada num ser humano integral, na qual elementos da Psicologia oferecem inúmeras contribuições. Sobre esse novo panorama, surge a Psicodermatologia, que segundo Azambuja (2000) representaria uma porta de entrada para estudos mais desafiantes, relacionados a esse novo ser humano, onde a pele aparece implicada com as diversas expressões das emoções humanas.

Assim, o corpo não poderia ser visto de forma reducionista, como apenas uma massa fisiológica. Autores como Cardoso Filho (2000) complementam tal idéia de integração, quando sugerem que o corpo ganha um forte significado, quando associado com as circunstâncias internas (orgânica) e externas (social). Por isso, podemos afirmar que o homem fala através do seu corpo, e do seu sintoma.

\section{O significado psicológico expresso pela pele}

A pele é um órgão exposto e visível. Portanto de fácil comunicação. Trata-se do maior órgão de percepção, no momento do nascimento, dessa forma, tornando-se o meio para o contato físico e, de imediata transmissão de sensações físicas e emocionais (MÜLLER, 2001). 
Pela nossa pele chegam os sinais do ambiente (calor, frio etc.), pois esta é a nossa fronteira de contato com o meio, transmitindo tais sinais aos centros do sistema nervoso. Capta também os sinais de nosso mundo interno (nossas emoções). Para Montagu (1988), a pele seria o espelho de como funciona nosso organismo: sua cor, textura, umidade, secura, e cada um de seus demais aspectos refletem nosso estado de ser, psicológico e também fisiológico".

Segundo Goleman e Gurim (1997), nossa pele pode ser comparada a um abrigo, o qual não tiramos para nada. Porém que muda conforme o nosso humor e o contexto em que estamos expostos. Praticamente todas as nossas emoções parecem ter impacto dinâmico na nossa pele. Portanto, em muitos casos, esta é a primeira a manifestar-se quando as aflições emocionais perturbam a nossa mente.

Müller (2001), já se utilizava da expressão neurodermite para exprimir alguns tipos de eczema, quando queria significar a sua origem nervosa.

Já para Dethlefsen e Dahlke (1997), a pele seria uma superfície que reflete, praticamente todos os órgãos internos. Dessa forma, qualquer distúrbio em um deles, seria imediatamente projetado na epiderme, em forma de arrepios, mudança na coloração, intensificação da sudorese, secura, ou até mesmo o aparecimento de eczemas.

A pele, além de demonstrar um estado físico interior e exterior de nossos órgãos, mostra também as correspondentes reações psíquicas na pele. Como exemplo, Silva (2002) cita o rubor (enrubescimento da face), encontrado, habitualmente, em pessoas que temem demonstrar seus sentimentos. Estes são evidenciados de forma involuntária pela pele. A sudorese intensa ou tremores são também citados pelo autor, pois, segundo o mesmo, exprimiriam um estado de ansiedade crônica.

Todavia, embora seja invisível, eletricidade na pele pode ser medida com equipamentos apropriados. Carl Gustav Jung (citado por DETHEFSEN e DAHLKE, 1997), investigou os reflexos galvânicos da pele, associados a suas experiências psicológicas. Atualmente é possível demonstrar, as contínuas e mais sutis alterações da condutividade elétrica da pele, bem como ampliá-las, a ponto de se poder utilizar para conhecer um pouco mais sobre as emoções das pessoas, associadas a determinados estímulos verbais ou visuais. Assim, pode-se pensar a pele como uma grande superfície de projeção.

A pele atua como um limite dentro-fora, eu e o mundo. Uma espécie de sistema de abrigo de nossa individualidade. Segundo Strausss "ao mesmo tempo em que nos protege, é a fachada que nos expõe" $(1989$, p. 1221).

Anzieu (1989) na mesma linha de raciocínio, refere-se a pele com um envelope do corpo, ao mesmo tempo, um envelope do psíquico.

Já Montagu (1988) enfatiza as correlações fisiopsicológicas envolvidas no contato. Ressalta a importância do toque e do tocas, para o desenvolvimento sadio do ser humano. 
Assim como os olhos, a pele também expressaria os estados emocionais, sendo capaz de uma auto-representação do ser. Além disso, ela refletiria conflitos e tensões presentes. (MEDANSKY; HANDLER; RIEDGE, 1981).

Silva (2002) nos orienta que, com frequiência, a história de vida dos pacientes portadores de doenças de pele, normalmente apresenta relatos de uma mãe inadequada, ou por superproteção, ou por ser rejeitadora. Isto nos leva a imaginar que, os conflitos intrapsíquicos, decorrentes de uma, podem ter grande importância na anamnese médica ou psicológica, quando do tratamento de complicações dermatológicas persistentes.

A doença de pele, em muitos casos, pode estar associada, a idéia de contágio ou de pouco asseio pessoal. Tais idéias implicam em um natural afastamento dos portadores dessas doenças.

A doença pode estar representando emoções que ainda o indivíduo não conseguiu resolver. Portanto as mesmas se apresentam como manifestações orgânicas. O sintoma é uma manifestação simbólica, como uma linguagem do que permaneceu oculto (AZULAY; AZULAY, 1997).

A doença, tem aspectos reprimidos, isso foi demonstrado por Groddeck (1989) e Jung (1985). Dessa forma, a compreensão da mesma implica necessariamente em se observar o paciente de forma integral, como um todo, um organismo psique-corpo que adoece.

\section{A Psicossomática da Dermatologia}

Medansky et al (1981) atribuiu a Erasmus Wilson, as ideias iniciais sobre a Psicossomática da Dermatologia. Ele, referiu em 1867, uma possível influência nervosa da pele com relação ao coçar e ao aparecimento de possíveis parasitoses, nem sempre confirmadas.

Damon, em 1868, publicou o trabalho Neuroses Cutâneas. Em 1895, Kaposi escreveu sobre as neuroses e afecções da pele e, em 1981, Bloch cunhou o termo neurodermatite. (AZULAY; AZULAY, 1997).

Spitz (1977) é um dos autores pioneiros, que apresentou extensivos trabalhos, que referam-se aos distúrbios iniciais nas relações mãe-bebê, como um entre os fatores causais da Demartite Atópica. Mais notadamente, se esses distúrbios ocorressem pouco antes do desenvolvimento da fala. Segundo o autor, uma combinação da imaturidade infantil e excitabilidade natural da criança, bem como da maneira imatura e hostil da mãe em gratificar as necessidades cutâneas táteis da criança, poderia ser responsável por desordens na pele na criança.

Outros trabalhos ressaltaram a manifestação de conflitos inconscientes através da pele, porém só recentemente, pelos idos de 1980, é que ressurgiu o interesse dos médicos dermatologistas, para estudo sobre a relação pele-psique. Segundo Koblenzer (1988) e Folks e Kinney (1992), isso 
aconteceu principalmente, à por conta da associação de estressores psicossociais, a uma baixa autoestima e, ao estigma social sofrido por parte de pacientes com problemas dermatológicos.

Esses mesmos autores realizaram uma revisão sistemática, de artigos publicados, sobre associação entre fatores psicológicos e dermatologia, entre 1980 e 1990, encontrando 418 citações. Portanto, um número de significativo de artigos foram encontrados sobre a temática. Entre os fatores psicológicos associados a dermatites estariam: pruridos, dermatites atópicas, hiperidrose, urticárias, rosáceas, alopécias e psoríases.

Müller (2001) referiu o vitiligo, que não foi citado pelos pesquisadores anteriores. Porém a autora demonstrou o o aparecimento do vitiligo, logo após uma situação de estresse emocional, portanto, ele também estaria associado aos fatores psicológicos.

Folks e Kinney (1992) referiram que muitas condições dermatológicas ainda estão sendo consideradas sob o ponto de vista da predisposição genética, de personalidade, ou de fatores biopsicossociais. As doenças da pele tendem a afetar, significativamente os relacionamentos, bem como a comunicação e, particularmente, o contato sexual dos indivíduos. Estudos nesta linha, mostram que estressores psicossociais, assim como perdas, desemprego, desajustamento interpessoal, e desordens de ansiedade, como hostilidade e baixa estima, estariam relacionados com condições dermatológicas.

As crianças cujas necessidades de estimulação tátil cutâneas não foram apropriadamente supridas na fase infantil, pode desenvolver uma natural tendência a regredir durante seu desenvolvimento emocional. Isso se daria em resposta ao estresse, com um consequente agravamento de sintomas físicos que podem estar relacionados à pele (KOBLENZER, 1983). Estes sintomas, segundo a autora, podem aparecer em forma de agressão, dentre outros.

A doença, para Koblenzer (1996), é normalmente precedida de um período de distúrbio emocional durante o qual, a pessoa sente-se não-apta para enfrentar. Esta falha nos mecanismos de enfrentamento psicológicos podem medidas emergenciais, de proteção, que se apresentam através do aparecimento de doenças, inclusive na pele.

A psiconeuroimunologia trouxe uma visão sistêmica do corpo humano, exigindo intervenções mais abrangentes. Portanto, no que se refere ao tratamento das doenças dermatológicas, além do tratamento médico alia-se, atualmente, segundo Folks e Kinney (1992), Moffaert (1992), Ginsburg (1996), Azulay e Azulay (1997), Goleman e Gurin (1997),: a) o tratamento psicoterápico; b) as técnicas de relaxamento, como hipnose e grupos de auto-ajuda; c) trabalho conjunto com um psicoterapeuta; d) uso de drogas psicotrópicas, mais especificamente, ansiolíticos e antidepressivos.

Tais intervenções exigem um tratamento individual e único para cada paciente. Como referem os autores Weinman, Petrie e Morris (1996), cada paciente tem uma representação do que seja a sua doença. Estes autores sustentam que, os doentes tem seus valores e crenças próprias sobre a causa, duração e, espectativa de cura da sua doença. Tais representações refletem a resposta cognitiva do 
paciente, para sua sintomatologia e, para quais as respostas emocionais serão processadas paralelamente à representação de doença.

Desta forma, segundo Müller (2001), percebe-se que a Psicodermatologia, quando estuda a relação pele-psiquê, busca romper com a visão unicausal da doença, em favor de um modelo mais holístico e multifatorial.

\section{Conclusões}

Mesmo diante de uma multiplicidade de estudos, que remetem a importância da integração mente e corpo, quanto às doenças dermatológicas, esses ambiguamente também nos apontam para a necessidade da construção de uma saúde menos dissociada e individualista, e mais voltada para uma visão de corpo e mente como unidade. Segundo Azambuja (2000), estudantes de Medicina são treinados para tratar doenças, observando antigos conceitos de saúde. Conforme o autor, encontra-se aí um paradoxo da Medicina: desejar chegar à saúde através do estudo das doenças, em vez de descobrir inicialmente a natureza que torna um corpo saudável.

Ressalta-se com tais reflexões, a necessidade dos profissionais da saúde, articulares a funcionalidade do orgânico, com a dinâmica do psíquico.

A psicossomática aparece como esse novo caminho, de apreender a conceber o homem como completo, como um todo (CARDOSO FILHO, 2000). Esse, é hoje, um novo ramo que vem se desenvolvendo à partir da Psicossomática: a Psiconeuroimunologia.

Trata-se de estudos que estão agregando inúmeras contribuições para a compreensão da interação mente e corpo, aproximando diversas áreas complementares como a Medicina e a Psicologia, por exemplo, as quais, assim como o psíquico e o físico, não devem ser encaradas separadamente.

Diversos autores nos sinalizam que o homem pode comunicar-se de diversas formas, e que a pele representa apenas uma dessas vias de comunicação. A pele pode representar, psicologicamente, um caminho para pedir ajuda, demandando uma necessidade de ser olhada, além dela mesma. Um olhar através da pele, pode representar um olhar além do corpo, além do que se mostra concreto, além do óbvio. E esse "olhar além", pode fazer toda a diferença, na compreensão de certas enfermidades, que desafiam os profissionais de saúde.

Este trabalho, lança aqui, pelo menos dois desafios aos profissionais quanto a promoção e prevenção da saúde. Primeiro o de lidar com o outro e conosco mesmos, na proximidade do contato e, segundo, o de concebermos uma reelaboração conceitual em nossa formação e quanto a nossa visão sobre o que vem a ser o humano. 
Id on Line Revista Multidisciplinar e de Psicologia

Id on Line Multidisciplinary and Psychology Journal

Assim, frente a tais reflexões e discussões, podemos pensar numa medicina e numa Psicologia que reconheçam o humano e sua doença, num sentido mais amplo, ou seja, numa Saúde, que deve tratar para além da doença e, consequentemente dos seus significados associados, do um ser humano inteiro.

\section{Referências}

AZAMBUJA, R. D. Dermatologia Integrativa: a pele em novo contexto. Anais brasileiro de Dermatologia, v. 75, n. 4, p. 393-420, 2000.

AZULAY, R; AZULAY, D. Dermatologia. Guanabara: Koogan, 1997.

BECHELLI, L. M.; CURBAN, G. V. Compêndio de dermatologia. São Paulo: Atheneu, 1988.

CARDOSO, F. A. A fala incontida no corpo. Revista da Associação Brasileira de Medicina Psicossomática, v. 4, p. 51-52, 2000.

DETHLEFSEN, T.; DAHLKE, R. A doença como caminho. São Paulo: Cultrix, 1997.

FITZPATRICK, T e Col. Dermatology. 3. ed. Nova York: International Edition, 1997.

FOLKS, D.; KINNEY, C. The role of psychological factors in dermatologic conditions. Psychosomatic, V. 33, n. 1, p. 42-54, 1992.

GINSBURG, I. The psychosocial impact of skin disease: an overview. Dermatologic clinics, v. 14, n. 3, p. 473 - 484, 1996.

GOLEMAN, D.; GURIN, J. Equilíbrio mente-corpo: como usar sua mente para uma saúde melhor. 3. ed. Rio de Janeiro: Campus, 1997.

GRODDECK, G. O homem e seu isso. São Paulo: Nova Fronteira, 1989.

JUNG, C. G. Fundamentos de Psicologia Analítica. 2. ed. Petrópolis, RJ: Vozes, 1985.

KOBLENZER, C. Psychosomatic concepts in dermatology. Archives of dermatology, v. 119, p. 501$512,1983$.

KOO, J; LEBWOHL, A. Psychodermatology: The mind and skin connection. American Academy of Fammily Physician, v. 11, n. 64, 2001.

MEDANSKY, R.; HANDLER, R.; RIEDGE, P. Dermatopsychosomatics: classification, physiology and therapeutic approaches. Journal of the American Academy of Dermatology, v. 5, n. 2, p. 125136, 1981. 
Id on Line Revista Multidisciplinar e de Psicologia

Id on Line Multidisciplinary and Psychology Journal

MOFFAERT, M. Psychodermatology: an overviem. Psychoterapy Psychosomatic, v. 58, p. 125136, 1992.

MONTAGU, A. Tocar o significado humano da pele. São Paulo: Summus, 1988.

MÜLLER, M. C. Um estudo psicossomático de pacientes com vitiligo numa abordagem analítica. 2001. 246 f. Tese (Doutorado em Psicologia) -- Pontifícia Universidade Católica de São Paulo, São Paulo.

SILVA, M. A D. Quem ama não adoece. São Paulo: Best Seller, 2002.

SPITZ, R. El primer año de vida del niño. Madrid: Aguilar, 1977.

STRAUSS, G. Skin disorders. Baltimore: Williams Wilkins, 1989.

WEINMAN, J.; PETRIE, K.; MORRIS, R. The illness perception questionaire: a new method for assessing the cognitive representation of illness. Psychology and health, v. 11, p. 431- 465, 1996.

\section{Como citar este artigo (Formato ABNT):}

BARROSO, M.L. Repercussões Psicossomáticas na epiderme humana. Id on Line Revista Multidisciplinar e de Psicologia, Julho de 2016, vol.10, n.30, p. 123-130. ISSN 1981-1179.

Recebido: $14 / 05 / 2016$

Aceito: 25/05/2016 